\title{
Magnetohydrodynamics of the Early Universe and the Evolution of Primordial Magnetic Fields*
}

\author{
D.T. Son ${ }^{\dagger}$ \\ Center for Theoretical Physics \\ Laboratory for Nuclear Science \\ and Department of Physics \\ Massachusetts Institute of Technology \\ Cambridge, Massachusetts 02139
}

(MIT-CTP-2724, hep-ph/9803412. March 1998, revised July 1998)

\begin{abstract}
We show that the decaying magnetohydrodynamic turbulence leads to a more rapid growth of the correlation length of a primordial magnetic field than that caused by the expansion of the Universe. As an example, we consider the magnetic fields created during the electroweak phase transition. The expansion of the universe alone would yield a correlation length at the present epoch of $1 \mathrm{AU}$, whereas we find that the correlation length is likely of order $100 \mathrm{AU}$, and cannot possibly be longer than $10^{4} \mathrm{AU}$ for non-helical fields. If the primordial field is strongly helical, the correlation length can be much larger, but we show that even in this case it cannot exceed 100 pc. All these estimates make it hard to believe that the observed galactic magnetic fields can result from the amplification of seed fields generated at the electroweak phase transition by the standard galactic dynamo.
\end{abstract}

Typeset using REVTEX

\footnotetext{
$\dagger$ Email address: son@ctp.mit.edu

${ }^{*}$ This work is supported in part by funds provided by the U.S. Department of Energy (D.O.E.) under cooperative research agreement \#DF-FC02-94ER40818.
} 


\section{INTRODUCTION}

Recently, considerable interest has been focused on the possibility that a primordial magnetic field may have been created at some early stage of the evolution of the Universe. While the existence of a weak widespread extragalactic chaotic magnetic field cannot be ruled out, almost the only place where such a primordial field may have left an observable imprint is in galaxies, many of which possess microgauss, kiloparsec scale fields that are thought to be the result of dynamo amplification of a weak seed field [1]. The idea that the seed for the galactic dynamo may be the field created in the very early Universe has inspired a number of works. For example, such fields may have appeared at the electroweak phase transition [2]. There are numerous other proposals involving physics at various scales (for a brief overview and further references, see [3]).

One feature shared by most particle-physics scenarios is the smallness of the correlation length of the magnetic field which results. Indeed, at the moment of creation, the correlation length is limited by the horizon radius. By pushing this moment to a very early stage in the history of the Universe, one makes the correlation length much smaller than it would be if the magnetic fields were created more recently, say, during proto-galactic contraction [4]. For example, the fields generated at the electroweak phase transition, when the horizon radius is about $1 \mathrm{~cm}$, would have a correlation length of at most about $10^{15} \mathrm{~cm}$ in the present Universe. Contraction of proto-galaxies is likely to reduce this length scale by 2 orders of magnitude, which gives $10^{13} \mathrm{~cm}$, or $1 \mathrm{AU}$, as the characteristic length scale of the seed field which the galactic dynamo is supposed to amplify. Realistically, this length scale is likely much smaller, since the correlation length at creation is typically much less than the horizon size円.

Because any primordial magnetic fields generated at early times (via particle physics occurring at, say, the electroweak scale) have such small correlation lengths, these fields are not attractive candidates to play the role of seed fields for the galactic dynamo, unless the correlation length is somehow increased. The best developed theory of magnetic amplification in galaxies, the mean-field dynamo [7], operates under the assumption that the magnetic field is smooth on the scale of turbulent motion of the interstellar gas, which is of order 100 pc. One may attempt to apply the mean-field dynamo theory for large-scale Fourier components of the chaotic magnetic field, neglecting the small-scale ones, however it is not obviously the correct procedure. That the magnetic field at very small scales exponentiates rapidly is a well known fact that poses a serious problem for the mean-field dynamo theory [8]. In the situation when the seed field itself resides at small scales, this problem is likely to become more severe.

In this paper, we will not address the question of how a seed field is created, whether at the electroweak phase transition or during some other early epoch. Our goal is to investigate

\footnotetext{
${ }^{1} \mathrm{~A}$ magnetic field created during or before inflation [5] may have a large correlation length, however the proposed inflationary models that could possibly generate a large-scale magnetic field may seem rather contrived [6]. We will not consider inflation-induced magnetic fields in this paper, but one should keep in mind this alternative.
} 
the possibility that magnetohydrodynamic (MHD) effects can lead to a substantial increase in the length scale of the magnetic field at the present epoch. We show that decaying MHD turbulence typically leads to a faster growth of the magnetic correlation length than one would expect from the expansion of the Universe alone. We estimate that in the case of magnetic fields generated at the electroweak phase transition, the enhancement factor is $10^{2}$, and the correlation length may reach $100 \mathrm{AU}$. If the primordial field has a large ChernSimons number, the enhancement factor may be much larger, but the correlation length cannot possibly exceed 100 pc today if the magnetic field comes from the electroweak epoch. Thus, although the magnetohydrodynamic effects we consider are of some help, they cannot increase the correlation length enough to make the electroweak generation of a primordial seed field a viable option. We also consider generating the seed field at the QCD phase transition. This may be a possibility, but is only viable if the bubble separation at the phase transition is very large.

This paper is organized as follows. In Sec. II we write the basic equation governing the MHD of the Universe. Sec. III is devoted to the decay of non-helical MHD turbulence, whereas Sec. $\mathbb{\square}$ describes the scaling laws of the decay of helical turbulence. Sec. $\nabla$ contains concluding remarks. The Appendix contains details about the EDQNM approximation used in our numerical simulation.

\section{THE MHD EQUATIONS}

To introduce notation, let us first write down the MHD equations for the early Universe. The MHD description applies since at interesting length scales the Universe is a good conductor. When the Universe expansion can be neglected (that is, when the evolution occurs on a time scale much smaller than the Hubble time), the MHD equations for a relativistic fluid have the form (for a derivation see, e.g., Ref. [9]),

$$
\begin{aligned}
\frac{\partial \mathbf{v}}{\partial t}+(\mathbf{v} \cdot \boldsymbol{\nabla}) \mathbf{v} & =-\frac{1}{\rho+p} \nabla\left(p+\frac{B^{2}}{2}\right)+\frac{1}{\rho+p}(\mathbf{B} \cdot \boldsymbol{\nabla}) \mathbf{B}+\nu \nabla^{2} \mathbf{v} \\
\frac{\partial \mathbf{B}}{\partial t} & =\boldsymbol{\nabla} \times(\mathbf{v} \times \mathbf{B})+\eta \nabla^{2} \mathbf{B}
\end{aligned}
$$

where $\mathbf{v}$ is the velocity of bulk fluid motion, $\mathbf{B}$ is the magnetic field, $\rho$ and $p$ are the density and pressure of the fluid, respectively. We assume that the bulk flow velocity is nonrelativistic, $v \ll 1$, although individual particles of the fluid move with the speed of light. The pressure can be eliminated from the equations by using the incompressibility condition, $\boldsymbol{\nabla} \cdot \mathbf{v}=0$ f . The kinematic viscosity $\nu$ and the resistivity $\eta$, both having the dimension of length in units in which $\hbar=c=1$, are determined by microscopic physics. One can define the kinematic and magnetic Reynolds numbers,

\footnotetext{
${ }^{2}$ The fluid can be considered incompressible if the Mach number, i.e. the ratio between $v$ and the sound speed, is much less than 1 . Since for ultra-relativistic fluids the speed of sound is $1 / \sqrt{3}$, this condition is essentially equivalent to $v \ll 1$.
} 


$$
\operatorname{Re}=\frac{v l}{\nu}, \quad \operatorname{Re}_{\mathrm{M}}=\frac{v l}{\eta}
$$

where $v$ and $l$ are the typical velocity and typical length scale of the fluid motion. As a rule, in the early Universe the magnetic Reynolds number is much larger than the kinematic one (i.e. the magnetic Prandtl number, defined as $\nu / \eta$, is large.) Hydrodynamic turbulence occurs when Re $\gg 1$. For example, at $T=100 \mathrm{GeV}, \nu \sim\left(\alpha T \log \frac{1}{\alpha}\right)^{-1}$, and $\eta \sim \frac{\alpha}{T} \log \frac{1}{\alpha}$. Taking $v \sim 10^{-1}, l \sim 10^{-2} a_{H}$, the two Reynolds numbers are $\operatorname{Re} \sim 10^{11}$ and $\operatorname{Re}_{\mathrm{M}} \sim 10^{17}$, i.e. very large.

Let us turn to the case of the expanding Universe. In the radiation-dominated epoch, neglecting the slow change of the number of degrees of freedom with temperature, the MHD equations are of almost the same form as Eqs. (11) [9],

$$
\begin{aligned}
\frac{\partial \mathbf{v}}{\partial \tau}+(\mathbf{v} \cdot \boldsymbol{\nabla}) \mathbf{v} & =-\boldsymbol{\nabla}\left(\tilde{p}+\frac{\tilde{B}^{2}}{2}\right)+(\tilde{\mathbf{B}} \cdot \nabla) \tilde{\mathbf{B}}+\tilde{\nu} \Delta \mathbf{v} \\
\frac{\partial \tilde{\mathbf{B}}}{\partial \tau} & =\boldsymbol{\nabla} \times(\mathbf{v} \times \tilde{\mathbf{B}})+\tilde{\eta} \Delta \tilde{\mathbf{B}}
\end{aligned}
$$

where $\tau=\int d t a^{-1}(t)$ is the conformal time, $\tilde{\mathbf{B}}=\mathbf{B} / \sqrt{\rho+p}$ is defined in such a way that the conservation of the magnetic flux during the expansion corresponds to the constancy of $\tilde{\mathbf{B}}$. The spatial derivative in Eq. (2) $\boldsymbol{\nabla}$ is taken with respect to the comoving coordinates. We have also defined $\tilde{\nu}=a^{-1} \nu$ and $\tilde{\eta}=a^{-1} \eta$, which are functions of time not only because of the factor $a^{-1}$ but also due to the temperature (and hence time) dependence of the viscosity and conductivity of the Universe. The formal coincidence of the MHD equations in the radiation-dominated Universe and in the non-expanding Universe allows us to discuss the evolution of the magnetic field in both cases from a common point of view.

\section{DECAY OF NON-HELICAL MHD TURBULENCE}

For definiteness, let us discuss the magnetic field generated during the electroweak phase transition, assuming the transition is first order [2,10]. It has been suggested in that after the phase transition, the cosmic fluid is in a state of turbulent motion, as parts of the fluid moving in front of different expanding bubbles collide [2]. An important point that has not been sufficiently emphasized in the literature is that until either another first-order phase transition happens, or gravitational instability begins to act, no additional stirring takes place and the turbulence will decay freely by the turbulent energy dissipation (see below.) As a consequence, turbulence does not continue forever, but should terminate at some point. Therefore, to establish the growth law of the magnetic correlation length, one has to study the free decay of MHD turbulence, and its termination.

Immediately after the phase transition, the characteristic velocity $v_{0}$ of the turbulence is that of the bubble walls. The correlation length of fluid velocity is the bubble separation

$l_{0}$. We will also assume the presence of an initial chaotic magnetic field that has the same correlation length as the velocity field and the same energy density as the turbulent bulk fluid motion. We rely here on the "equipartition" hypothesis, which states that any small seed magnetic field (which may have been created during the phase transition [2] or come 
from the hypercharge field created at some earlier epoch [10]) will be enhanced by turbulent convection until the magnetic energy is equal to the kinetic energy of the bulk fluid motion, $\tilde{B}_{0}^{2} \sim v_{0}^{2}$. Whether equipartition hypothesis is valid is a controversial issue. It is known that an arbitrarily weak magnetic field is exponentially enhanced by turbulence when $\mathrm{Re}_{\mathrm{M}}$ is larger than some critical value (of order 100, which is easily satisfied in cosmological conditions), but it is unclear what is the saturation level of the magnetic energy. However, it is very unlikely that we underestimate the magnetic field by relying on equipartition. As even this magnetic field will prove insufficient, we will not worry whether we are making an overestimate. (The values of $v_{0}$ and $B_{0}$ mentioned above correspond to the integral scale, i.e. the largest scale of turbulence, which dominates the energy.)

We will also presume for the present that the mean density of the Chern-Simons number is vanishing. This is the case if the initial condition is parity symmetric in the statistical sense. We discuss the case of nonzero Chern-Simons number, and why it is different, in a later section of this paper.

\section{A. Non-expanding Universe: scaling laws}

Let us first consider the simpler case, in which the expansion of the Universe can be neglected. This corresponds to the first step of the evolution, when the time passed from the creation of the magnetic field is smaller than the Hubble time. We will first make a simple argument of how the correlation length grows with time, and then discuss the assumptions behind this argument. The mechanism for the growth is selective decay: modes with larger wavenumbers decay faster than those whose wavenumbers are small. Thus, at increasing time only modes with smaller and smaller wavenumbers survive. Let us assume that at some time $t$ only modes with length scale larger than $l$ survive. The modes that are about to decay, i.e. those at the scale $l$, dominate the kinetic and magnetic energies. Denote the typical value of $v$ and $B$ of these modes as $v_{l}$ and $B_{l}$. From the equipartition hypothesis, one has $\tilde{B}_{l} \sim v_{l}$. Let us find the dependence of $v_{l}$ and $B_{l}$ on $l$. If we assume that modes with wavelengths larger than $l$ remain the same during the decay of shorter modes, the configuration of $v$ and $B$ at moment $t$ is essentially the initial configuration, smeared out over the length scale $l$. At the time moment $t$, the value of $v$ and $B$ at a point in space is roughly the average of the initial $v$ and $B$ over the sphere of radius $l$ around this point. Assuming that $v$ and $B$, at $t=0$, is not correlated over lengths larger than $l_{0}$ (which is certainly true if $l_{0}$ is the Hubble size of the Universe at this time), the average is over $\left(l / l_{0}\right)^{3}$ patches with random $v$ and $B$. Therefore, the order of magnitude of $v$ and $B$ at the moment $t$ is

$$
v_{l} \sim v_{0}\left(\frac{l}{l_{0}}\right)^{-3 / 2} \quad B_{l} \sim B_{0}\left(\frac{l}{l_{0}}\right)^{-3 / 2}
$$

The characteristic time for the decay of modes with length scale $l$ is the eddy turnover time,

$$
\tau_{l} \sim \frac{l}{v_{l}}
$$

Notice that the decay time (41) does not depend on any viscosity and resistivity. This is the consequence of the turbulent cascade: during the time $\tau_{l}$ the energy is transfered from modes 
with length scale $l$ to $l / 2$, then it is cascaded to $l / 4, l / 8$ etc. with faster and faster rate until the viscosities enter the scene at some small scale $l_{\text {diss }}$ (which is called the dissipation scale). Due to the cascade, the energy (both kinetic and magnetic) is dissipated at a much faster rate than it would be without turbulence. The length scale $l_{\text {diss }}$ does not enter any of our formulae, as we are interested in the much longer length scales at which the cascade begins; all that matters is that dissipation occurs at some very small length scale, terminating the cascade.

Since $l$ is the smallest wavelength that survives at the moment $t$, its lifetime must be comparable to $t$. Solving $\tau_{l} \sim t$ using Eq. (B), one finds that the correlation length scales with time as,

$$
l(t) \sim\left(\frac{t}{t_{0}}\right)^{2 / 5} l_{0}
$$

where $t_{0}=l_{0} / v_{0}$ is the eddy turnover time at $t=0$. From Eqs. (3).5) and the fact that the energy is proportional to $v^{2}$ and $B^{2}$, we find that the latter decays as $t^{-6 / 5}$. It is worth noting that all our arguments leading to these scaling laws are also valid for decaying hydrodynamic (not MHD) turbulence. Indeed, the $t^{-6 / 5}$ decay of the turbulent energy is known in fluid dynamics as Saffman's law [11, 12].

The implicit assumption behind our derivation of the $t^{2 / 5}$ law (5) is that the spectrum at low $k$ remains unmodified during the decay of high- $k$ modes. Such an assumption is essential for deriving Eq. (3) (called "volume averaging" elsewhere.) The validity of this assumption may seem uncertain, since inverse cascade, i.e. the process of transferring energy from high to low modes happens under some conditions in MHD. In [13, 3], it was suggested that inverse cascade is a generic feature of MHD turbulence and could greatly enhance the correlation length of the magnetic field (although, coincidentally, the scaling law for the growth of the correlation function obtained in [13,3] is the same as our Eq. (5).) What we want to argue here, to support the derived scaling laws, is that the inverse cascade of magnetic energy required a special condition to be met, namely, the average density of the Chern-Simons number must be non-zero. That the conditions for the inverse cascade

are rather restrictive is a well known fact in plasma physics: previous studies 14 16 , have identified these conditions to be either a non-zero Chern-Simons number density of the magnetic field, or a non-helical turbulent flow (see also the discussion below.) Since we do not expect the bulk fluid flow to be helical, the only chance to have an inverse cascade is if the magnetic field has non-zero Chern-Simons number, which is not the case considered in this Section. We have also checked that there is indeed no inverse cascade in the non-helical MHD turbulence decay by simulating it using the eddy-damped quasi-normal Markovian (EDQNM) approximation. Our simulation will be described in the next subsection.

\section{B. Numerical simulation of decaying non-helical turbulence in a non-expanding Universe}

To verify the absence of the inverse cascade, as well as to check our scaling laws, we use the EDQNM approximation for numerical simualations. A full discussion of the merits and shortcomings of the EDQNM approximation can be found in Refs. 112,14, here we will only 
note that this approximation is a member in a family of closure schemes, which are similar to the Hartree-Fock approximation in field theory. The EDQNM approximation has been found very successful in reproducing qualitative features of hydrodynamics and MHD as the Kolmogorov spectrum of driven hydrodynamic turbulence, the inverse cascade in MHD (under the above-mentioned conditions), etc. One should be aware that it is unclear how the EDQNM approximation can be systematically improved. However, it should be adequate to answer our qualitative questions.

In the closure schemes, the turbulence is characterized by two functions $E_{k}$ and $M_{k}$, which are the spectra of kinetic and magnetic energy (in the case of helical flow and helical magnetic fields, one should introduce two more functions of $k$.) The full set of EDQNM equations are quite complicated [15]. In the Appendix, we reproduce the equations for the case of non-helical turbulence. As the initial condition at $t=0$, we choose the initial kinetic and magnetic spectra to have the same functional form,

$$
E_{k}=0.9 \frac{4}{\pi} \frac{k^{2}}{\left(k^{2}+1\right)^{2}}, \quad M_{k}=0.1 \frac{4}{\pi} \frac{k^{2}}{\left(k^{2}+1\right)^{2}}
$$

The $k^{2}$ behavior of $E_{k}$ and $M_{k}$ at low $k$ 's comes from the assumption that the initial state consists of uncorrelated eddies?. In the initial condition (6), the magnetic energy constitutes $10 \%$ of the initial total energy at $t=0$, which is equal to 1 . In choosing the initial magnetic energy to be small compare to the total energy, we obtain as a bonus the possibility of checking whether equipartition is reached during the decaying turbulence. We choose $\nu=$ $\eta=10^{-3}$, which correspond to the Reynolds numbers of order $10^{3}$ at $t=0$. This is much smaller than the cosmological Reynolds numbers, but large enough for the physics at the integral scale to be insensitive of the exact values of Re and $R e_{M}$. We use logarithmic discretization in the $k$ space, with 4 points per octave (we have checked that doubling the number of points per octave does not alter the result in any substantial way), and the simplest first-order time-update algorithm.

The main results are summarized in the Fig. (11-3). In Fig. 1, the spectra of kinematic and magnetic degrees of freedom are plotted at $t=0$ and $t=100$ (measured in units of the eddy turnover time at $t=0)$. We see that the low- $k$ part of the spectrum is not modified by turbulent decay, which means that there is no inverse energy cascade. It is interesting to note that, at $t=100$, in the high- $k$ part of the spectrum the magnetic energy exceeds the kinetic energy, and there is a range of $k$ where the Iroshnikov-Kraichnan spectrum $E_{k} \sim M_{k} \sim k^{-3 / 2}$ is observed. Both features have been seen before in the simulation of forced (not decaying)

\footnotetext{
${ }^{3}$ To see that, one could use the following argument. The typical wavelength in the initial state is $k_{0} \sim l_{0}^{-1}$. If one throws away all modes with momentum larger than some $k \ll k_{0}$, this is equivalent to smearing out the velocity and the magnetic field over the distance of $k_{0}^{-1}$. As discussed before, if $v$ and $B$ do not correlate over lengths larger than $k_{0}^{-1}$, the average takes both down by a factor of $\left(k / k_{0}\right)^{3 / 2}$. The energy, which is proportional to $v^{2}$ and $B^{2}$, is down by a factor of $\left(k / k_{0}\right)^{3}$. Compare this with the case of an energy spectrum with $k^{2}$ behavior at low $k$, in which throwing away modes with energy larger than $k$ makes the energy of remaining modes proportional to $\int_{0}^{k} d k k^{2} \sim k^{3}$.
} 
turbulence [15]. In Fig. 2, the typical wavelengths of kinetic and magnetic modes, which are defined as

$$
k=\frac{\int d p E_{p}}{\int d p p^{-1} E_{p}}, \quad k_{\mathrm{M}}=\frac{\int d p M_{p}}{\int d p p^{-1} M_{p}}
$$

are plotted versus time from $t=0$ to 1000 . Both $k(t)$ and $k_{\mathrm{M}}(t)$ decreases at large $t$ and their time dependence agrees very well with our prediction, $k \sim t^{-2 / 5}$. The ratio $k_{\mathrm{M}}(t) / k(t)$ approaches a finite value (about 2) at large $t$. We conclude that our simple arguments reproduce correctly the behavior of the correlation length in the non-helical turbulence decay. In Fig. 3, we plot the dependence of the total kinetic and magnetic energy as functions of time. The long-time behavior is well described by Saffman's law $E \sim t^{-6 / 5}$. Notice that the magnetic energy grows for a few eddy turnover periods before decaying. Apparently, equipartition is at work here: at first some energy is pumped from the kinetic motion into the magnetic field. Equipartition is never fully realized, but the ratio of the magnetic energy to the kinetic energy approaches a value closed to 1 (namely, 0.8) in our simulation. Therefore, we have seen that our qualitative arguments (the most important being the lack of inverse cascade) agree well with the numerical simulation.

\section{Expanding Universe}

So far we have considered the case of a non-expanding Universe. In the radiationdominated Universe, the correlation length, in comoving coordinates, grows as $\tau^{2 / 5}$, where $\tau$ is the conformal time. Since $\tau \sim T^{-1}$ ( $T$ being the temperature), we conclude that in this case the growth of the correlation length follows the $T^{-2 / 5}$ law (not counting the trivial redshift).

In the real situation we have a hybrid of the two cases. If at $t=0$ the eddy turnover time $t_{0} \sim l_{0} / v_{0}$ is smaller than the age of the Universe, there will be a substantial period of time when the expansion of the Universe can be neglected, that is, when $0<t \lesssim t_{H}$. The expansion factor for the length is then $\left(t_{H} v_{0} / l_{0}\right)^{2 / 5}$. When $t \gtrsim t_{H}$, one has to take into account the expansion, and the increase in the correlation length (not counting the expansion) is $\left(T_{\mathrm{EW}} / T_{0}\right)^{2 / 5}$, where $T_{0}$ is the temperature of the Universe when the fluid flow becomes non-turbulent (we determine $T_{0}$ below.) Therefore, the final correlation length at the present epoch is,

$$
l_{\text {now }}=l_{0} \cdot\left(\frac{t_{H} v_{0}}{l_{0}}\right)^{2 / 5} \cdot\left(\frac{T_{\mathrm{EW}}}{T_{0}}\right)^{2 / 5} \cdot \frac{T_{\mathrm{EW}}}{T_{\mathrm{now}}},
$$

where the last factor comes from the expansion of the Universe. Putting in numbers, using for $T_{0}$ the temperature of $e^{+} e^{-}$annihilation (which is of order $m_{e}$ ), using $v_{0} \sim 0.1$ and $l_{0} \sim 10^{-2} a_{H}$, one finds $l_{\text {now }} \sim 100$ AU. This scale is larger than the scale one would have if the only mechanism of dissipation of magnetic energy is resistive diffusion (about 1 AU [17]), but it is still too small to be a direct seed for the mean-field dynamo. The situation is better if one takes larger values of $v_{0}$ and $l_{0}$. However, even in the extreme case when the magnetic field is initially correlated on the horizon size and its initial energy density is comparable to that of the whole Universe, the correlation length is still less than $10^{4} \mathrm{AU}$. 
Let us check that $T_{0}$ is the temperature at which $e^{+} e^{-}$annihilation occurs, which is to say that below this temperature, turbulence ceases, and therefore so does the enhancement of the magnetic correlation length. To this end, we need to compute the Reynolds number Re before and after the $e^{+} e^{-}$annihilation. At $T_{e^{+} e^{-}} \sim 1 \mathrm{MeV}$, using Eq. (7) and replacing $T_{\text {now }}$ by $T_{e^{+} e^{-}}$, one finds the correlation length $l \sim 10^{19} \mathrm{GeV}^{-1}$. The velocity at $T_{0}$ is

$$
v \sim v_{0} \cdot\left(\frac{t_{H} v_{0}}{l_{0}}\right)^{-3 / 5} \cdot\left(\frac{T_{\mathrm{EW}}}{T_{0}}\right)^{-3 / 5} \sim 10^{-5}
$$

and the viscosity is $\nu \sim\left(\alpha^{2} T\right)^{-1} \sim 10^{7} \mathrm{GeV}^{-1}$. The kinematic Reynolds number is then $10^{5}$.

After $e^{+} e^{-}$annihilation, however, the cosmic fluid becomes much more viscous. This is the result of the dramatic drop of the density of charged particles, which leads to a very long mean free path for photons, which now dominate the viscosity. The viscosity jumps by the photon-to-baryon ratio, $10^{10}$, and the Reynolds number becomes small. Therefore, $e^{+} e^{-}$ annihilation is the moment when turbulence terminates/. After this moment, the magnetic field is frozen into the essentially non-moving fluid and the correlation length increases only by the expansion of the Universe, as we assumed in deriving Eq. (7).

\section{DECAY OF HELICAL TURBULENCE}

Let us now turn to the case when the original magnetic field is helical. Here helicity refers to the Abelian Chern-Simons number, $N_{\mathrm{CS}}=\int d \mathbf{x} \mathbf{A} \cdot \mathbf{B}$, which is called the magnetic helicity in plasma physics. The situation when the initial field has a non-vanishing ChernSimons number is not entirely hypothetical, since some mechanisms indeed generate such helical fields [10]. However, our interest will be mostly in finding out the largest length scale one could possibly get from a magnetic field from the electroweak epoch.

It is a well known fact in plasma physics that the Chern-Simons number is an approximately conserved quantity of MHD turbulence [14]. If the fluid is an ideal conductor, the conservation of $N_{\mathrm{CS}}$ is exact. This can be seen from the geometrical interpretation of the Chern-Simons number: $N_{\mathrm{CS}}$ is proportional to the number of links of the magnetic field lines. When $\eta=0$, the latter are frozen in the moving fluid, and the number of links cannot change with time. At nonzero $\eta$, the conservation of $N_{\mathrm{CS}}$ is less obvious, since another conserved quantity, the energy $E=\frac{1}{2} \int d \mathbf{x}\left((\rho+p) v^{2}+B^{2}\right)$, dissipates at a finite rate at arbitrarily small $\nu$ and $\eta$. The smallness of the dissipation of $N_{\mathrm{CS}}$ can be explained in the following (non-rigorous) way, which relies only on the difference between the dimensions of $E$ and $N_{\mathrm{CS}}$. Let us write down the equations for the dissipation of the energy and the Chern-Simons number, which can be derived from Eq. (11) [14],

\footnotetext{
${ }^{4}$ It is sometimes, based on the results of [18], argued that the large diffusion length of photons leads to damping of the magnetic field, similar to the Silk damping of density fluctuations. However, Ref. [18] deals with the damping of $M H D$ waves but not the background magnetic field on top of which these waves propagate (see also the analysis in Ref. [19].)
} 


$$
\begin{aligned}
\dot{E} & =-\int d \mathbf{x}\left(\nu(\rho+p)(\boldsymbol{\nabla} \times \mathbf{v})^{2}+\eta(\boldsymbol{\nabla} \times \mathbf{B})^{2}\right) \\
\dot{N}_{\mathrm{CS}} & =-2 \eta \int d \mathbf{x} \mathbf{B} \cdot(\boldsymbol{\nabla} \times \mathbf{B})
\end{aligned}
$$

Let us compare the order of magnitude of the right hand sides of Eqs. (8,9), assuming for simplicity $\nu \sim \eta$ and equipartition, $\rho v^{2} \sim B^{2}$. If both $E$ and $N_{\mathrm{CS}}$ are dominated by the largest length scale of turbulence (the integral scale) $l$, then $N_{\mathrm{CS}} / E \sim l$. On the other hand, the dissipation of both energy and the Chern-Simons number occurs at the dissipation scale $l_{\text {diss }}$. Since $l_{\text {diss }}$ is much smaller than $l$ (by a power of the Reynolds numbers), we conclude that $\dot{N}_{\mathrm{CS}} / \dot{E} \ll N_{\mathrm{CS}} / E$, which means that the dissipation rate of the Chern-Simons number goes to zero in the limit of small viscosity and conductivity, in contrast to the energy which dissipates with a finite rate in this limit. When the Reynolds numbers are large, one can neglect the dissipation of the Chern-Simons number (but not of the energy.)

The conservation of $N_{\mathrm{CS}}$ has an important consequence for the evolution of the magnetic field. When $N_{\mathrm{CS}}$ is non-vanishing, the short-scale modes are not simply washed out during the decay: their magnetic helicity must be transfered to the long-scale ones. Along with the magnetic helicity, some magnetic energy is also saved from turbulent decay. This process is known as the inverse cascade [14,15]. The coupling with kinetic motion ensures that a part of the energy cascaded to large scales also goes to the kinetic energy of fluid motion, maintaining equipartition. We will assume maximal helicity, i.e. $\mathbf{A} \cdot \mathbf{B}$ has the same sign in all space, so the magnetic helicity density is proportional to $A B \sim l B^{2}$. Since this quantity is conserved during the decay of the turbulence, the field strength should scale with the correlation length as

$$
B_{l} \sim B_{0}\left(\frac{l}{l_{0}}\right)^{-1 / 2}
$$

This law replaces Eq. (3). This corresponds to "line averaging" that gives a much larger amplitude of the magnetic field than the "volume averaging [20]. However, our averaging is not postulated, but is a dynamical effect which occurs only in the helical case. An argument similar to the one used in the non-helical case now gives the following decay law for helical MHD turbulence:

$$
l \sim l_{0}\left(\frac{t}{t_{0}}\right)^{2 / 3}
$$

which replaces the formula (5). Therefore, the correlation length grows faster if the initial field configuration is helical. Obviously, this comes from a slower decay of the magnetic field, which implies a larger equipartition velocity and hence a shorter relaxation time compared to the non-helical case.

\footnotetext{
${ }^{5}$ Inverse cascade occurs also in two-dimensional hydrodynamics and MHD. In both cases, the conserved quantities governing the inverse cascade, the enstrophy and the mean square magnetic potential [14], are positively defined, so the inverse cascade does not requires special initial conditions.
} 
To see what is the largest magnetic length one can get, let us assume that at $T=100$ $\mathrm{GeV}$ one has $l_{0} \sim a_{H}$ and $v_{0} \sim 1$. Assuming the turbulence has all decayed at $T_{0}$, the correlation length at $T=T_{0}$ is

$$
l=l_{0}\left(\frac{T_{\mathrm{EW}}}{T_{0}}\right)^{5 / 3}
$$

The velocity now scales as $v \sim\left(T_{\mathrm{EW}} / T_{0}\right)^{-1 / 3}$. In the helical case, with our choice of parameters, the turbulence in fact survives beyond $e^{+} e^{-}$annihilation. When $T \ll m_{e}$, the viscosity of the Universe is mostly due to photons and is given by

$$
\nu \sim \frac{1}{n_{e} \sigma_{T}} \sim \frac{m_{e}^{2}}{\eta_{B} \alpha T^{3}}
$$

where $n_{e}$ is the electron density, $\eta_{B}$ is the baryon-to-photon ratio, and $\sigma_{T} \sim \alpha m_{e}^{-2}$ is the Thompson cross section. One sees that the kinematic Reynolds number drops below 1 at $T_{0} \sim 100 \mathrm{eV}$. Therefore, the correlation length is now

$$
l \sim l_{0} \cdot\left(\frac{T_{\mathrm{EW}}}{T_{0}}\right)^{5 / 3} \cdot\left(\frac{T_{0}}{T_{\mathrm{now}}}\right) \sim 100 \mathrm{pc}
$$

Since the collapse of proto-galaxies will reduce this correlation length by a factor of 100 , the galactic seed field will be correlated on a scale of order $1 \mathrm{pc}$, still much smaller than the scale of the turbulent motion of the interstellar gas which we recall is of order 100 pc. Therefore, the presence of helicity in the initial magnetic field strongly enhances the magnetic correlation length, but still cannot make it large enough to be the seed for the mean-field galactic dynamo.

\section{CONCLUSION}

In this paper we have considered the behavior of the correlation length of the primordial magnetic field, if such a field is generated in the early Universe. We found the decay law of the MHD turbulence, which is responsible for a substantial increase of the correlation length. We also observed a qualitative difference between the cases of non-helical and helical initial magnetic field. Taking the case in which the magnetic field is generated during the electroweak phase transition as an example, we find that if the magnetic field is not helical the factor gained from MHD is about $10^{2}$ and today the field may be correlated at scales as large as $100 \mathrm{AU}$. If by some chance the field is helical, the enhancement factor is much larger, but the final correlation length cannot exceed $100 \mathrm{pc}$, corresponding to $1 \mathrm{pc}$ after proto-galactic collapse.

Let us note some uncertainties remaining in our estimation. (In general, addressing these issues would lead to less optimistic values of the enhancement factor.) First, it is not at all clear whether in MHD turbulence the magnetic energy reaches equipartition with the kinetic energy of bulk fluid motion. While semi-analytical calculations (including our simulation) favor equipartition [15], some numerical results indicate that the mean magnetic energy density remains small and concentrated at scales shorter than the largest turbulence scale 
[16]. If the latter remains true at very high Reynolds number, the magnetic field will be smaller after the electroweak phase transition and the correlation length at present will be smaller than we have estimated.

The second factor is neutrino diffusion. After the electroweak phase transition, neutrinos are the particles with the longest mean free path. There is a certain time interval when neutrinos are still not decoupled from the physics at the turbulence scale, but their diffusion length is so large that the neutrino contribution to viscosity makes the Reynolds number to drop below 1. During this time, there is no turbulence and the magnetic field is frozen. After the neutrinos decouple from the fluid motion at the turbulence scale, magnetic stress leads to restoration of turbulence, and the magnetic length may continue to grow. The overall effect is some reduction in the estimate of the final magnetic correlation length.

It would be nice to end this paper on a more positive note, and to this end we turn now to investigating the QCD phase transition, which occurs later than the electroweak transition and so may yield a longer correlation length. If the QCD phase transition is first order, it will introduce fresh turbulence stronger than the decaying electroweak turbulence. The amplitude and correlation length of the magnetic field will be determined by the QCD turbulence. If fields were regenerated at the QCD transition on length scales of order of the horizon, the length scale it would have today is very large, since the horizon size at the QCD phase transition is much larger than at the electroweak epoch. Even with no enhancement from MHD effects, the expansion of the universe yields a magnetic correlation length of order 1 pc. It can be estimated that the turbulence will survive till at least matter-radiation decoupling, at which the correlation length is is of order $1 \mathrm{kpc}$ in the non-helical case and $100 \mathrm{kpc}$ if the field is helical. However, a magnetic field correlated on the horizon size is not expected to be produced at the QCD phase transition, and these estimates are too optimistic. Instead, the initial length scale must be of the order of the bubble spacing at the end of the phase transition (which is the natural scale of turbulence). Bubble spacings larger than $100 \mathrm{~cm}$ are unlikely [21] and may have problem with standard big bang nucleosynthesis [22]. A bubble spacing smaller than $100 \mathrm{~cm}$ again implies a very small correlation length at the present epoch. If this constraint is respected, our analysis of the QCD case must end pessimistically, as in the electroweak case. However, one cannot completely rule out the possibility of a very large bubble spacing, which leads to a magnetic field correlated on a long enough length scale to be of interest. A nonstandard evolution in which mixing after the phase transition occurs rapidly by hydrodynamic flows instead of slowly by diffusion or nonstandard nucleosynthesis may be required in this case.

Finally, although in this paper we presented the smallness of the magnetic correlation length as undesirable and tried to overcome it by invoking MHD turbulence, there may exist a non-standard dynamo mechanism where a small-scale seed gives rise to a large-scale

magnetic field (see, e.g., [23]). This possibility is, however, outside the scope of the present paper.

\section{ACKNOWLEDGMENTS}

The author thanks R. Jackiw, S.-Y. Pi, E. Vishniac, and especially J. Berges and K. Rajagopal for stimulating discussions. He thanks H. Kurki-Suonio and K. Subramanian for 
comments on the manuscript. This work is supported in part by funds provided by the U.S. Department of Energy (D.O.E.) under cooperative research agreement \#DF-FC0294ER40818.

\section{APPENDIX A: THE EDQNM APPROXIMATION}

As mentioned in the main text, in closure schemes, the non-helical turbulence is characterized by the kinetic and magnetic energy spectra, $E_{k}$ and $M_{k}$, defined so that the total kinetic and magnetic energies are $\int_{0}^{\infty} d k E_{k}$ and $\int_{0}^{\infty} d k M_{k}$, respectively (we assume isotropy, so the spectra depend only on the absolute value of $\mathbf{k}$.) The evolution of the spectra, in the EDQNM approximation, are described by the following equations,

$$
\begin{aligned}
\left(\partial_{t}+2 \nu k^{2}\right) E_{k}= & \int d p d q \theta_{k p q}\left[\frac{1}{q}\left(x y+z^{3}\right)\left(k^{2} E_{p} E_{q}-p^{2} E_{q} E_{k}\right)-\frac{p^{2}}{q} z\left(1-y^{2}\right) M_{q} E_{k}+\right. \\
& \left.+\frac{k^{2}}{q} z\left(1-y^{2}\right) M_{p} M_{q}\right] \\
\left(\partial_{t}+2 \eta k^{2}\right) M_{k}= & \int d p d q \theta_{k p q}\left[\frac{k^{3}}{p q}(1+x y z) M_{p} E_{q}-\frac{k p}{q}\left(1-y^{2}\right) E_{q} M_{k}-\right. \\
& \left.-\frac{k^{2}}{q} z\left(1-y^{2}\right) M_{q} M_{k}\right]
\end{aligned}
$$

which are the non-helical version of the full set of EDQNM equations that can be found in Ref. [15]. In Eq. (A1), the integration over $p$ and $q$ is perform in the region where $k, p$ and $q$ form a triangle; $x, y$ and $z$ are the cosines of the angles of this triangle,

$$
x=\frac{p^{2}+q^{2}-k^{2}}{2 p q}, \quad y=\frac{q^{2}+k^{2}-p^{2}}{2 q k}, \quad z=\frac{k^{2}+p^{2}-q^{2}}{2 k p}
$$

and $\theta_{k p q}$ is defined as

$$
\theta_{k p q}=\frac{1-\mathrm{e}^{-\mu_{k p q} t}}{\mu_{k p q}}, \quad \mu_{k p q}=\mu_{k}+\mu_{p}+\mu_{q}
$$

where

$$
\mu_{k}=(\nu+\eta) k^{2}+C_{s}\left(\int_{0}^{k} d q q^{2}\left(E_{q}+M_{q}\right)\right)^{1 / 2}+C_{a} k\left(\int_{0}^{k} d q M_{q}\right)^{1 / 2}
$$

$C_{s}$ and $C_{a}$ are phenomenological constants, the exact values of which are not qualitatively important. Following [15], we choose $C_{s}=0.26$ and $C_{a}=1 / \sqrt{3}$. 


\section{REFERENCES}

[1] P.P. Kronberg, Rep. Prog. Phys. 57, 325 (1994).

[2] G. Baym, D. Bödeker, and L. McLerran, Phys. Rev. D53, 662 (1996); G. Sigl, A. Olinto, and K. Jedamzik, Phys. Rev. D55, 4582 (1997).

[3] P. Olesen, NATO ASI Series (Plenum) B366, 159 (1998).

[4] R.M. Kurlsrud et al., Astroph. J. 480, 481 (1997).

[5] M.S. Turner and L.M. Widrow, Phys. Rev. D 37, 2743 (1988).

[6] B. Ratra, Astrophys. J. 391, L1 (1992); Phys. Rev. D 451913 (1992).

[7] A.A Ruzmaikin, A.M. Shukurov, and D.D. Sokoloff, Magnetic Fields of Galaxies, Kluwer, Dodrecht, 1988.

[8] R.M. Kulsrud and S.W. Anderson, Astroph. J., 396, 606 (1992).

[9] A. Brandenburg, K. Enqvist, and P. Olesen, Phys. Rev. D54 1291 (1996).

[10] M. Giovannini and M. Shaposhnikov, Phys. Rev. D57 2186 (1998); Phys. Rev. Lett, 80, 22 (1998).

[11] P.G. Saffman, Phys. Fluids, 10, 1349 (1967).

[12] M. Lesieur, Turbulence in Fluids, 3d ed., Kluwer, Dordrecht, 1997.

[13] P. Olesen, Phys. Lett. B398, 321 (1997);

[14] D. Biskamp, Nonlinear Magnetohydrodynamics, Cambridge University Press, Cambridge, 1993.

[15] A. Pouquet, U. Frisch, and J. Léorat, J. Fluid Mech., 77, 321 (1976); J. Léorat, A. Pouquet, and U. Frisch, J. Fluid Mech. 104, 419 (1981).

[16] M. Meneguzzi, U. Frisch, and A. Pouquet, Phys. Rev. Lett. 47, 1060 (1981); S. Kida, S. Yanase, and J. Mizushima, Phys. Fluids A3, 457 (1991).

[17] B. Cheng, A.V. Olinto, Phys. Rev. D50, 2421 (1994).

[18] K. Jedamzik, V. Katalinic, and A. Olinto, Phys. Rev. D57, 3264 (1998).

[19] K. Subramanian and J.D. Barrow, astro-ph/9712083.

[20] See, e.g. K. Dimopoulos and A.-C. Davis, Phys. Lett. B390, 87 (1997).

[21] J. Ignatius et al, Phys. Rev. D 50, 3738 (1994).

[22] H. Kurki-Suonio et al., Phys. Rev. D38, 1091 (1988); D. Thomas et al., Astroph. J. 430, 291 (1994).

[23] B. Chandran, Astroph. J. 492, 179 (1998). 


\section{FIGURES}

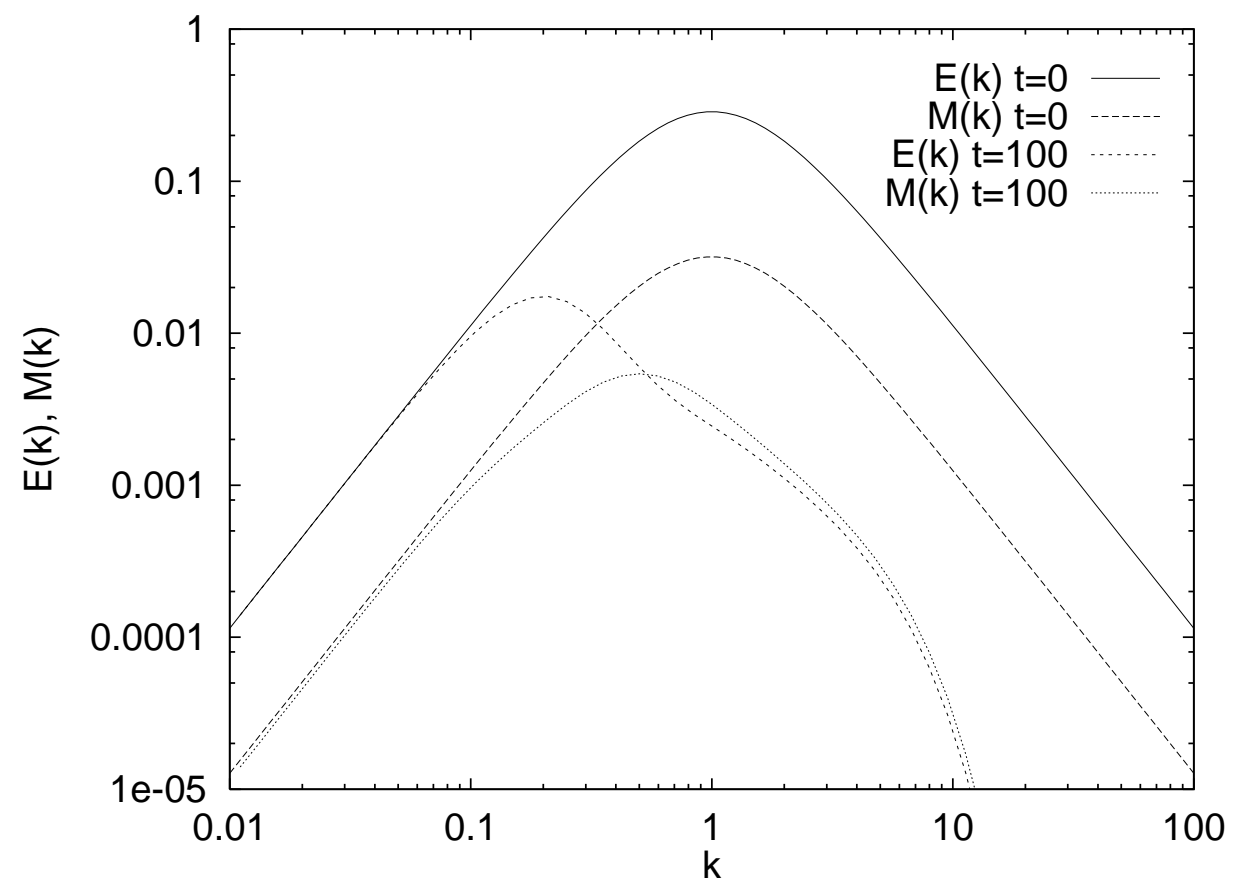

FIG. 1. The kinetic and magnetic spectra at $t=0$ and $t=100$

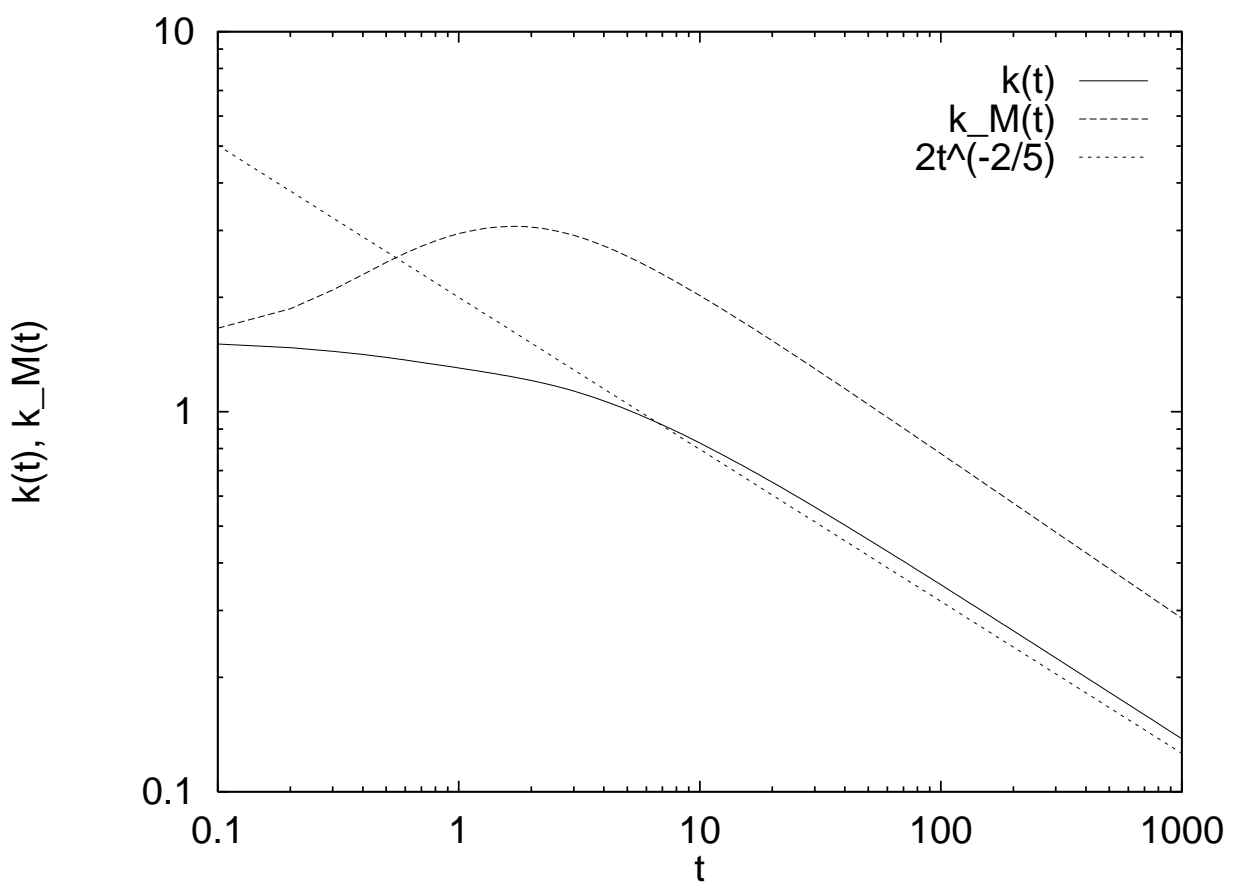

FIG. 2. Time evolution of the typical wavelength 


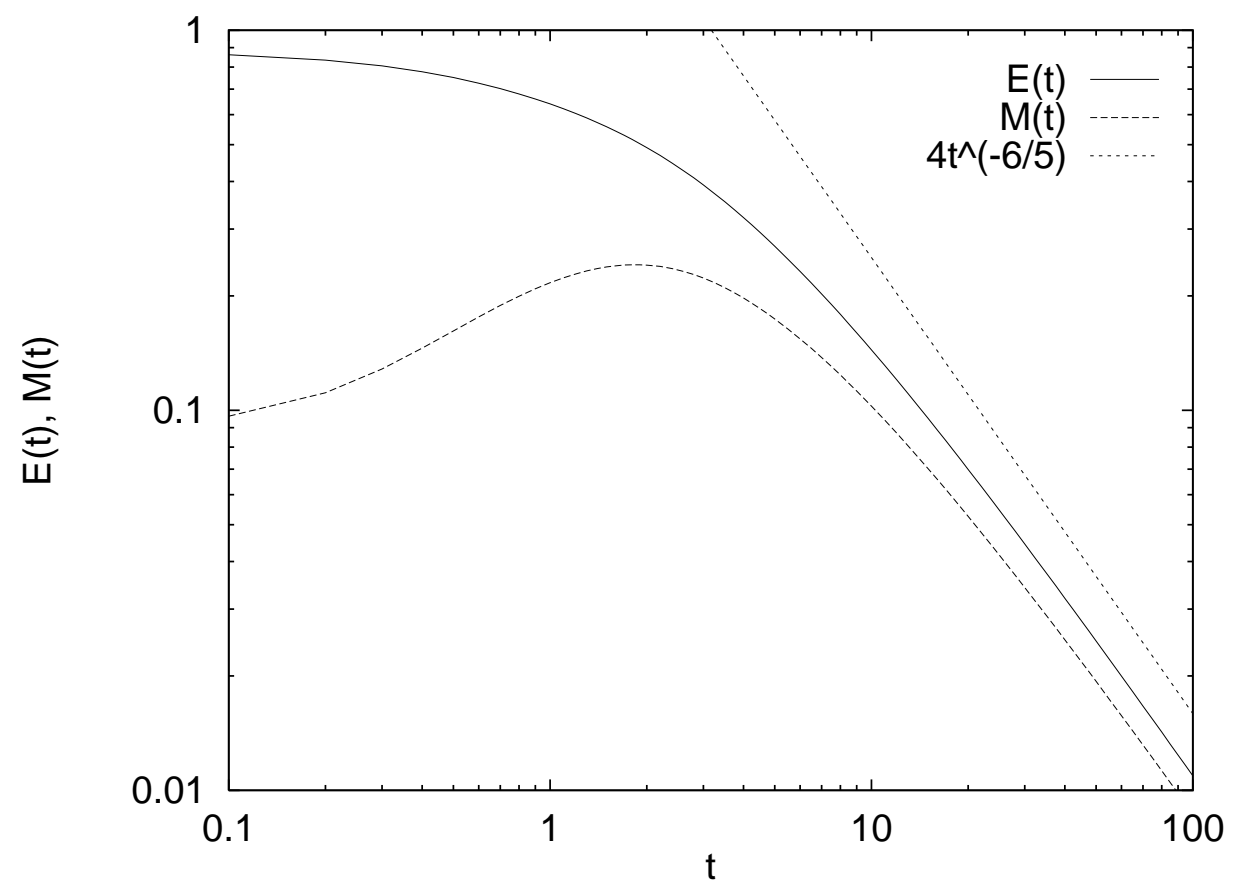

FIG. 3. Time evolution of the total kinetic and magnetic energy 